\title{
Impact Analysis of Collaborative Platforms - The Case of SPIKE
}

\author{
Christian Broser, Christoph Fritsch, Oliver Gmelch, \\ Günther Pernul
Department of Information Systems, University of Regensburg, 93040 Regensburg, Germany
(e-mail: $\{$ christian.broser, christoph.fritsch, oliver.gmelch, guenther.pernul\}@wiwi.uni-regensburg.de).

\begin{abstract}
Today's companies are under considerable strain. Several events or developments such as the global economic crises or the ongoing globalization process contribute to this situation. Meanwhile, however, companies have realized that the concept of virtual organizations represents an efficient measure to counter the current challenges. More and more companies make use of this concept supported by technologies like collaboration platforms. However, the application of such collaboration platforms brings along some changes for organizations in doing their business. For instance, interaction between people, who could not get in touch before, is thereby fostered in particular. Employees of different organizations, even across countries' boundaries, can set up new relationships due to the deployment of collaboration platforms. This paper analyses the impact of collaboration platforms and their effects on the daily business of organizations.
\end{abstract}

Keywords: collaboration; networked enterprise; impact analysis; virtual alliance

\section{INTRODUCTION}

The proceeding globalization, increasing market pressure and shorter innovation cycles are only an extract of challenges today's organizations face. Additionally, the current global economic crisis increases the pressure on the companies. To counter those challenges, organizations have to rethink their ways of doing business in order to stay successful and achieve sustainable competitiveness. In this context, Virtual Organizations (VO, also called virtual alliances or virtual enterprises) appear as promising choice where core competencies, resources and skills of the involved partners are joined.

Breaking up entrenched business structures to enable flexible project structures and performing business through collaborative value chains across companies' borders with anybody, anywhere, anytime - regardless of underlying information technology infrastructures and business processes - represents a promising way as identified by van Heck and Vervest (2007). Recent surveys by AT\&T Corp. (2008) and by Eid, T. and Gartner Research (2007) back this trend and predict a significant increase of Virtual Organizations in the forthcoming years. The technology used behind such VOs is provided by collaboration platforms. Those platforms enable communication in different ways such as email, instant messaging or wiki systems between the alliance partners. Thereby such collaborations can be run completely virtual; physical interaction by e.g. going abroad to visit a partner company is no more necessary. VOs can be set up much faster and operated more efficiently. Thus, also the human and social aspects play an essential role. For instance, new relationships and partnerships between employees - or generally between people - can be evolved easier. Organizations' or countries' boundaries are no more observed as real obstacles.

A further advantage is that companies are thereby enabled to enter new, previously inaccessible, markets because of the concentrated force behind the collaboration. In essence, small and medium-sized enterprise can profit by this model. In most economies, small and mediumsized enterprises (SME) represent the biggest fraction of companies. For instance, in the European Union, SMEs comprise $99 \%$ of all companies (Thompson (2008)). For them, flexible collaboration with partners is of particular interest because today, the key to economic success is flexible and fast reaction to market opportunities which still is limited by organizations' cumbersome business structures. As already mentioned, the trend to VOs supported by Collaboration Platforms affects also social aspects or human aspects, respectively. Already in 1994, Rosabeth Moss Kanter stated that "successful collaborations build and improve a collaborative advantage by first acknowledging and then effectively managing the human aspects of (...) alliances" (Kanter (1994)).

This paper analyses the impact of collaboration platforms on existing and potential upcoming aspects. The EUfunded research project SPIKE $^{1}$ will serve as an example, from which potential factors and aspects are derived. This paper therefore is intended to serve as a case study, taking into account results like those published by Hersh (2001) or Kile (2001), whereas deeper investigation is left up to future research. The remainder of the paper is outlined as follows: In section 2 we present the components and

\footnotetext{
1 http://www.spike-project.eu
} 
functionality of collaborative platforms. Subsequently, the SPIKE project will be introduced in the following section. Section 4 derives an impact analysis of aspects arising from SPIKE. Finally, section 5 gives an outlook on social aspects in conjunction with collaboration platforms and concludes this paper.

\section{COLLABORATIVE PLATFORMS}

\subsection{Global Competition}

Rising competition between enterprises on local as well as on regional and global scale demands for higher agility and flexibility of companies. Especially the impacts of the current financial crisis and the accompanying losses in total order quantities intensify this competition. As a result of this trend, many companies increasingly focus on their key competences, meaning the business areas they have superior knowledge of and are especially successful in. However, this specialization goes alongside an increased number of customers in the narrowed business area and a decreased variety of provided services per company. This in turn requires tight interplay with other, likewise specialized business partners along the value creation chain.

This new collaborative approach requires companies to move from fairly stable and long lasting business partnerships to an open network of short-term, possibly even spontaneous cooperations. Across these rapidly formed networks of participants, business is conducted with anyone, independently of each participant's location and despite different business processes and computer systems spanning organizational hierarchies and different organizational structures.

The social aspects on the organizational and interpersonal level arising from those cooperations are laid out in section 4. However, to allow for such a flexible business network, digital technologies play a fundamental role in these business networks and comprehensive tool support is indispensable. Currently, several approaches exist to satisfy these requirements which are shortly presented in the following.

\subsection{Current Approaches \& Tool Support}

Software and tools supporting business networks and cooperations can be found in different fields and several flavours. The most well-known umbrella term for such systems is groupware. Other terms are CSCW (Computersupported Cooperative Work) systems or collaboration software. All these systems and tools have in common that they support business networks, Virtual Organizations and collaborative projects in one way or another.

Ellis et al. (1991) presented an approach to classify computational support for collaboration. The $3 \mathrm{C}$ collaboration model (see figure 1) developed by Borghoff and Schlichter (2000) is derived from that article and is based on the idea that communication, cooperation, and coordination are the major challenges for members in a collaborative group. Basically, the $3 \mathrm{C}$ model is equivalent to the Clover model by Laurillau and Nigay (2002) as well but focuses on cooperation instead of production. The $3 \mathrm{C}$ approach

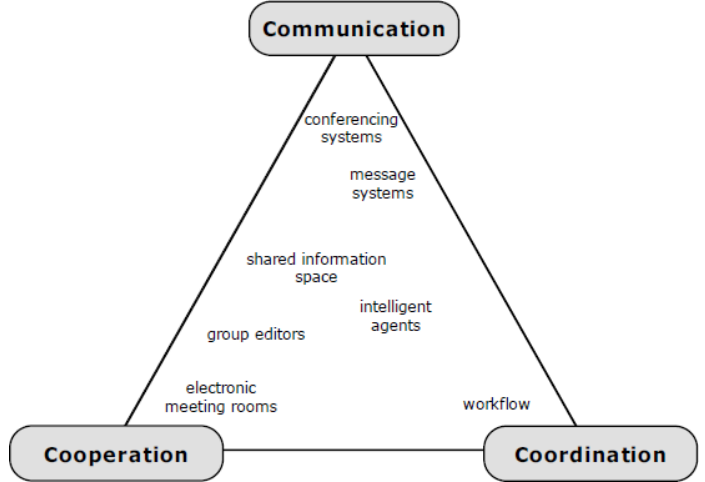

Fig. 1. Classification of Groupware Systems (Borghoff and Schlichter (2000))

has been known for a long time and widely used in the literature to classify collaborative tools (Borghoff and Schlichter (2000)) or by Bandinelli et al. (1996) to improve the computational support of software processes.

Currently available groupware tools can be assigned to one of the dimensions shown in figure 1 as most of them have a rather clear focus. Still, most of them support tasks in the remaining two dimensions in one way or another.

- Communication involves the exchange of messages and the negotiation of commitments and is mostly supported via email, instant messaging, discussion lists, video/phone conferences and the like. Characteristic products focusing on that dimension are Microsoft Exchange or Novell Groupwise.

- Coordination enables people, activities, and resources to be managed so as to resolve conflicts and facilitate communication and cooperation. Coordination tools support resource, staff and project management, scheduling, and monitoring. Tools such as OpenGroupware, eGroupware or dotProject belong to this group.

- Cooperation is the joint production of members of a group within a shared space, generating and manipulating cooperation objects to complete tasks. Supportive tools typically assist via joint document storage and repositories, cross-company usage of applications or wiki- and notes keeping systems. Microsoft Sharepoint, IBM Lotus Notes Domino and Moodle are mainly classified in this dimension.

All in all, all three dimensions are currently supported well by different tools. However, no single tool exists that supports all three dimensions to a satisfactory extent. Despite their separation for analytic purposes, communication, coordination, and cooperation should not be considered isolated from each other as there is constant interplay between them. One noticeable fact is that the three dimensions and their tool support focus on standardizing and supporting social contacts between team members, possibly even cross-organizational.

\subsection{A new Business Network Approach}

The technologies presented in the previous section have facilitated improvements and fundamental changes in the way organizations interact with one another and prepared 
the basic possibility to form alliances. However, in a crossorganizational setting, current approaches are suitable to only a limited extent. Organizations are moving, or forced to move, from today's relatively stable and slowevolving business networks with only a few, long-term partnerships to an open digital platform where business is conducted under a short-term perspective with varying partners and across several organizations, irrespective of different domains and home countries. Virtual Organizations and Networked Enterprises are born and strongly rely on demanding flexibility and scalability. Participants typically take part not in a single but in a number of VOs. These digital networks require tight interactions between businesses and individuals and can expose unexpected behavioural properties of the individual actors. As each business network participant has specific capabilities and each team member in such a VO specializes on his/her key competencies, this approach very well matches the industrialization approach in the manufacturing industry during the second half of the 18th century.

Regarding the tool support, companies preparing for participation in VOs must modularize their production systems by decomposing their systems and flexibly regrouping the resulting elements for mixing and matching the companies' responsibility in the current project. Hoogeweegen et al. (1999) developed a method to design modular business networks and to optimize the allocation of tasks in a business network based on modularity principles. Current groupware systems, however, do not yet allow for the desired degree of flexibility and integrated standard software is usually not flexible enough to cope with these requirements.

Ideally, collaboration platforms exist, on which users can freely move and interact as long as the platform provider allows to do so. Barabasi (2003) demonstrated that such platforms show a strong network effect, meaning that the more users (companies, individuals) the platform attracts, the more useful the network becomes and the more difficult it becomes for participants to switch, whilst the likelihood that the user moves to another network decreases.

\subsection{Business Opportunities}

The major aim of the depicted business network approach is to gain new business opportunities through extended flexibility for companies and individual employees. Smart business networks promise to allow for "rapidly pick, plug, and play" business processes and to immediately meet specific objectives such as to react to a customer order or an unexpected situation (van Heck and Vervest (2007)). Smart businesses become smart by capturing a valuable position in a network of companies and leveraging this position to prepare as many links to other nodes as possible. Several examples show that these business networks can respond much more agile and flexible and provide better results than any individual participant can while acting as a single company.

In this context, the European Union identified the potential of this approach, targeting the information society and the digital economy in the action plan i2010 (Reding (2005)). One of their major concerns is that a global digital economy in all its facets would be of benefit for

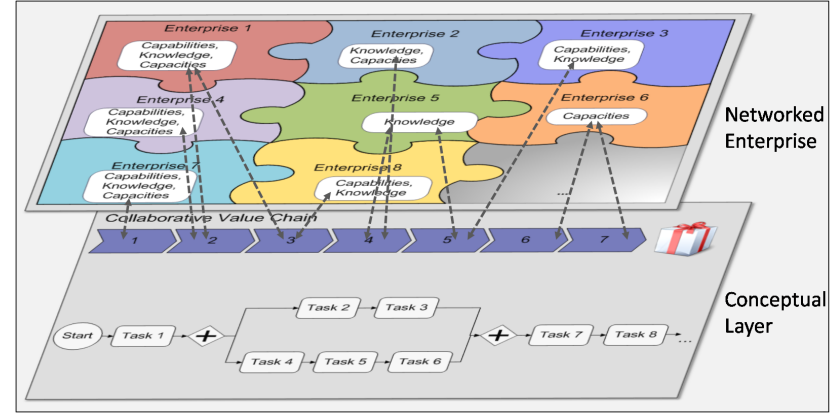

Fig. 2. Conceptualization of a networked enterprise

European economy and its SMEs in particular. All kinds of companies can boost their efficiency and especially networks of highly specialized SMEs can make use of new business opportunities as they can now compete with large internationally operating organizations.

\section{THE SPIKE RESEARCH PROJECT}

\subsection{Common SPIKE Characteristics}

The goal of the SPIKE project is to deliver a software platform for the easy and fast setup of shortterm, project-based and loosely coupled business alliances among partner organizations. "Loosely coupled" in the SPIKE context means that there is no need for further pre-established technical or organizational relationships between the prospective partnering organizations. SPIKE basically targets two main organizational objectives:

- Outsourcing parts of the value chain to business partners (and vice versa, offering such parts in form of services).

- Enabling collaboration between members of participating organizations through ad-hoc created as well as predefined business processes.

\subsection{SPIKE Architecture}

The SPIKE project includes a three-layered architecture of a business alliance. For simplification reasons and to address the social aspects arising through collaboration platforms, presentation of this architecture is split into two individual figures. Figure 2 shows the Networked Enterprise layer where different companies exhibit and provide their particular knowledge, expertise, capabilities, capacities, and resources. As it may be necessary to outsource parts of the value chain to business partners, companies of different sizes from diverse sectors, specialized in varying areas, join forces and build virtual business alliances.

The second layer on the bottom of figure 2, the SPIKE Conceptual Layer, is the conceptualization of the business alliance and its goals. Usually, a collaborative project starts with a common goal of all alliance partners, only accomplishable by joining forces. To reach that goal, a highlevel strategy has to be agreed upon by all involved parties, which in later steps is further refined into a detailed business process specification. In the SPIKE context, the business process specification is performed via Business 
Process Modelling Notation (BPMN) ${ }^{2}$. The modelling of the collaboration project's workflow on an abstract level is done outside the SPIKE platform, employing existing tools such as Eclipse BPMN Modeler ${ }^{3}$, Adonis ${ }^{4}$ or Visual Paradigm ${ }^{5}$. All these BPMN tools allow transforming the graphical BPMN model to a Business Process Execution Language (BPEL) ${ }^{6}$ process which is then input to the SPIKE platform. Modelling on an abstract level means that the service providers that are responsible for carrying out given tasks in the workflow are not yet assigned in the BPMN model, but dynamically assigned by the SPIKE platform at runtime of the resulting BPEL process. Within the SPIKE portal, the BPEL representation of the collaboration project's workflow is then further refined, the actual service providers that support a given task in the workflow are statically assigned or can be specified via required and semantically specified capabilities and goals. This allows for dynamic selection of the actual service instance at runtime of the workflow, considering different specified criteria such as availability or costs per service request.

The SPIKE solution encompasses a semantically enriched service-oriented infrastructure including a virtual semantic service bus for workflow control and handling and transformation of messages. At the enterprise interface level, a collaborative process portal approach is followed, capturing the user's working context and seamlessly transmitting it to other applications and services in the alliance. This also allows for integration of legacy systems via tailored portlets and connectors. Special focus is given to the security and identity management issues involved. The solution includes an easy-to-administer security infrastructure for the business alliance. Additionally, federated identity management and administration of digital user identities and privileges across organizational boundaries is another major issue. A strong focus on the security and identity management aspects is necessary because of the focus on "project-based" collaborations, meaning that alliance partners possibly are competing simultaneously in other projects not part of the alliance.

\section{IMPACT ANALYSIS ARISING FROM SPIKE}

\subsection{Support of Collaboration Phases}

Collaboration platforms change the way companies organize themselves internally and also impose the need to open their processes to external partners, embedded in larger value chain networks. This section is devoted to raising awareness about the impacts on social interactions of said collaboration platforms, influenced by the experiences made during the development of the SPIKE collaboration platform.

Foremost, as can be seen from figure 3, collaborations usually are characterized by a set of individual states they

2 OMG - Business Process Modeling Notation (BPMN), http://www.omg.org/docs/formal/09-01-03.pdf

3 http://www.eclipse.org/bpmn/

4 http://www.boc-group.com/index.jsp?file=WP_582571cc1ed802de. 46e381.f59775478f.-7f17\&lg=en

5 http://www.visual-paradigm.com/

6 OASIS - Web Services Business Process Execution Language

Version 2.0, http://docs.oasis-open.org/wsbpel/2.0/wsbpel-v2.0.pdf

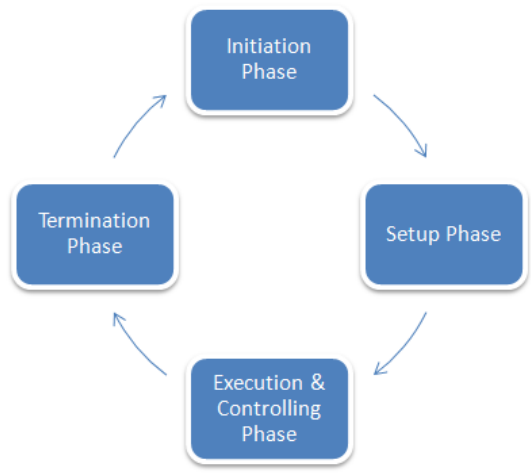

Fig. 3. Collaboration phases

subsequently traverse, as also identified by AT\&T Corp. (2008) and adopted for this presentation:

(1) Initiation phase. This phase consists of searching for suitable partners in a networked enterprise, as well as settling of all legal aspects, carried out by upper management.

(2) Setup phase, consisting of the definition of the actual processes to follow during a collaboration as well as the implementation of mechanisms to grant access to the systems in use to other partners. In the setup phase, it is up to specialized domain experts to adapt the system to the special needs of a specific collaboration project.

(3) Execution and controlling phase, containing instances of the processes defined in the previous phase as well as usage of all services alongside permanent monitoring of the current collaboration conditions. In this phase, task execution is characterized by involvement of staff into relatively easy tasks which can be carried out by rather low-skilled employees. In contrast, monitoring and exception handling duties, which impose a high level of responsibility, require company management employees with higher skill level.

(4) Termination phase, consisting of removal of access to all systems, potentially also dissolving from other network partners. This phase involves both ending the contractual situation by management staff as well as removing access to the remote systems previously used within a VO.

In the past, a lack of dedicated tools supporting the aforementioned phases of collaborations lead to a multitude of individual human interactions, taking place via various information channels, i.e. emails, phone calls, fax messages, etc. With the upcome of dedicated collaboration tools such as the aforementioned collaboration platform SPIKE, a process of strong formalization takes places during collaboration definition and even more during collaboration execution time. This shift towards the actual service integration is also depicted in figure 4, which shows the second part of the SPIKE architecture. Due to space restrictions, this and the subsequent sections of this paper discuss three main social implications imposed by the usage of collaboration platforms and experienced during the development of the SPIKE project. 


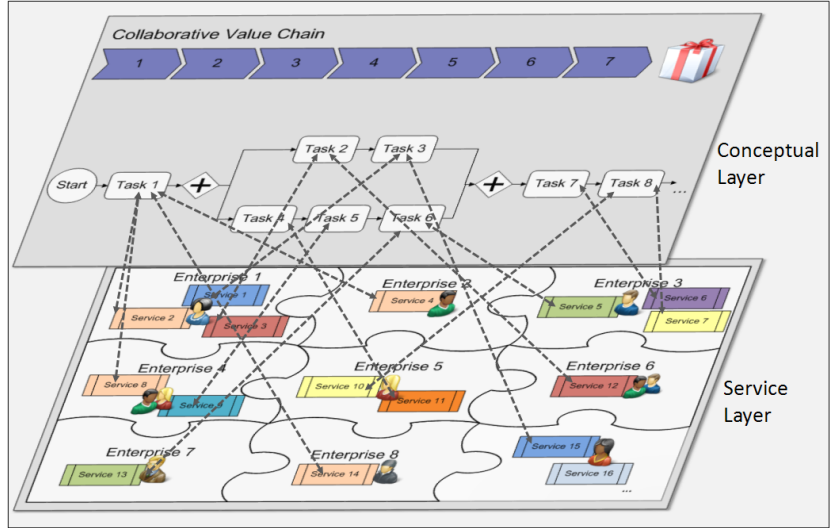

Fig. 4. Formalization during collaboration definition

\subsection{Process Orientation}

Historically, due to a lack of collaboration-aware software, intra-company workflows were mainly driven by human interactions, allowing for flexible adjustments to frequently changing conditions on the one hand. On the other hand, however, this huge level of human interaction heavily relied on the availability of specially crafted workflow managers supervising all activities performed in such environments. Also, the overall workforce is required to always manually keep track of the most recent manifestation of every process an individual user is involved in as well as to synchronize with all predecessors and successors concerning task execution. Consequently, a high level of social interaction is required in order to carry out the envisioned duties. The aforementioned formalization of collaboration projects, however, implies a change of attitude and a huge shift towards automated process control, which can be compared with the shift from individually handcrafted vehicles to assembly line production in the car manufacturing industry, which started in the early 1920's.

Using modelling languages such as BPMN and interpreting the results in collaboration platforms such as SPIKE allows for an orchestration of individual activities into process chains, thus optimizing the overall productivity of both individuals involved in single processes as well as the companies employing these technologies on a larger scale. This, however, implies that the aforementioned high level of social skills in order to organize workflows between individual employees, which was previously a high priority criterion for the employer, is no longer as important since the communication and synchronization overhead can be reduced to a bare minimum as a consequence of the introduction of collaboration-aware software support. This is especially true when considering the execution phase of a collaboration, where previously-defined workflows are interpreted by the workflow engine, taking care of all synchronization and data transfer aspects. On the other hand, especially the initiation phase still bears a strong demand for people showing a high level of social skills in order to make the business contacts and to find and convince potential partners for a networked enterprise. Also, escalation processes necessary during the execution and controlling phase show a high demand for social skills in order to rule out any conflicts arising within a collaboration.

With this in mind, the usage of collaboration-aware software tools can help to support a company's workforce during the actual task execution and to lower the burdens imposed by synchronization and data transfer between the individuals involved in said tasks. During collaboration initiation, setup and monitoring phases, however, still highly-skilled people are necessary in order to keep a collaboration operating smoothly. Furthermore, it is up to the company leaders to identify and take on business opportunities arising from the formation of value chain networks.

\subsection{Dissolving of Company Boundaries}

Globalization, shorter innovation cycles, and increased competition represent only an extract of challenges today's organizations, in essence SMEs, face. In this context the networked enterprise as new paradigm of innovative, progressive and successful organizations arises.

While both large organizations as well as SMEs have realized that business alliances with other companies, even competitors, can be very beneficial and possibly inevitable, particularly SMEs have an increased interest in collaboration platforms in order to boost their efficiency as well as make use of new business opportunities to compete with large internationally operating organizations. Today, the key to economic success is flexible and fast reaction to market opportunities. This situation thus requires enabling of workflows for operation across multiple domains by providing digital services to other parties without great efforts.

Again, from an end user's perspective, the introduction of collaboration-aware software tools manifests itself in a reduction of social interaction as well as a change of attitude of a company's employees, caused by the need to dissolve company boundaries and the integration into larger value chain networks. Company managers no longer can solely be held responsible for the outcomes of their internally-lead projects, but in contrast will have to take responsibility also for the selection of partners and the results of the larger value chain networks.

Furthermore, the automatic selection of a specific service instance during runtime based on predefined criteria imposes great challenges to companies to optimize their service quality. With the availability of an automatically generated service execution history of previous service usage such as execution time or availability and the resulting high level of transparency, high pressure is generated on potential partners in a VO to optimize their own level of service delivery as much as possible in order to remain an attractive partner within VOs. Even though this may appear as a threat to the individual company, on a global scale this process helps to improve overall quality within a virtual organization by relying on the best partners available.

\subsection{Impact on Globalization}

The aforementioned dissolving of company boundaries, followed by the integration of external partners in larger value chain networks, also bears large chances for organizations not yet affiliated with other networked enterprises. As the entry barriers for joining value chain networks and for creating innovative product ideas, are relatively low, new enterprises may appear on the market quickly and 
easily. For instance, new players on the market can be attracted by the ability to adopt to new business models that distinguish their offerings from those of their competitors. Likewise, the opportunity to outsource tasks to alliance members with a better cost structure in an easy and fast way bears great chances for companies located in developing countries.

This is also supported by the open-source characteristic of the SPIKE platform, where new platform providers can take the existing solution as a basement and adjust it to their own special needs. In this context, it is noteworthy that the usage of SPIKE is not necessarily inclined to opening up company boundaries. Instead, SPIKE can also be used for internal projects in order to strengthen the individual company's competitive situation.

Another area of competitiveness is that very often SMEs do not have possibilities to offer their services globally or sometimes not even nationwide, due to the nature of service or lack of "interface personnel". Global business usually requires a marketing and supply network and to be visible and efficient, often investments are needed that small businesses do not have resources for. SPIKE will help in many levels to address that problem by formalizing the supply interactions and offering reference scenarios for reuse.

Putting the idea of the freely available SPIKE platform in a global context, non-discriminatory access to participation in networked enterprises can embed developing countries within future global economical developments, meaning that developing countries can benefit from globalization encountered in global economy.

\section{CONCLUSION \& OUTLOOK}

Tightened global competition in general and the current financial crisis in particular force organizations to move from today's rather few and stable long-term business partnerships to a more flexible and agile approach. Networked Enterprises and Virtual Organizations, in which business is conducted with a short-term perspective and with many different partners, marks such a new business network approach. These versatile networks span different companies even across frontiers and have a considerable impact on involved people and their interpersonal relationships.

In this paper we illustrate how and to what extent current and future cooperation approaches influence social aspects and interpersonal relationships. On the one hand, participants in Virtual Organizations have to focus on their key competences and specialize on particular services they perform in very high quality. This is redolent of the industrialization in the manufacturing industries and restricts individuals' freedom. On the other hand, however, people acting in those Virtual Organizations have the chance and are obliged to interact with many different co-workers with different backgrounds and across varying companies. Furthermore, we give a short review of current tool support in the field of collaboration software and lay out the SPIKE platform as a representative for software supporting the business network approach.

Future work in the SPIKE project covers further development of the technical components, where it will be of particular interest how the current SPIKE solution for a collaboration platform is accepted by participants and prospective users. Furthermore, we will further investigate how SPIKE as an electronic collaboration platform influences and possibly encourages the way organizations and personnel interact in an inter-enterprise and inter-domain setting.

\section{ACKNOWLEDGEMENTS}

The research leading to these results is receiving funding from the European Community's 7th Framework Programme under grant agreement no. 217098. The content of this publication is the sole responsibility of the authors and in no way represents the view of the European Commission or its services.

\section{REFERENCES} $\begin{array}{cccc}\text { AT\&T Corp. } & \text { (2008). } & \begin{array}{l}\text { Collaboration } \\ \text { borders. }\end{array} & \text { Technical } \\ \text { report. } & \text { URL }\end{array}$ http: //www . corp. att.com/emea/docs/s5 collaboration_eng.pdf.

Bandinelli, S., Nitto, E.D., and Fuggetta, A. (1996). Supporting Cooperation in the SPADE-1 Environment. IEEE Transactions on Software Engineering, 22(12), 841-865.

Barabasi, A.L. (2003). Linked: How Everything Is Connected to Everything Else and What It Means. Plume, reissue edition.

Borghoff, U.M. and Schlichter, J.H. (2000). ComputerSupported Cooperative Work: Introduction to Distributed Applications. Springer-Verlag New York, Inc., Secaucus, NJ, USA.

Eid, T. and Gartner Research (2007). Web conferencing and team collaboration software, worldwide, 2006-2011.

Ellis, C.A., Gibbs, S.J., and Rein, G. (1991). Groupware: Some Issues and Experiences. Communications of the $A C M$, Volume 34 , Issue 1, 39-58.

Hersh, M. (2001). Technology change, technology transfer and ethics. In P. Kopacek (ed.), Social Stability: The Challenge of Technology Development (SWIIS '01, IFAC), 63-68. Pergamon Press.

Hoogeweegen, M., Teunissen, W., Vervest, P., and Wagenaar, R. (1999). Modular Network Design: Using Information and Communication Technology to Allocate Production Tasks in a Virtual Organization. Decision Sciences 30 (4), 1073-1103.

Kanter, R.M. (1994). Collaborative advantage - the art of alliances. Hardvard Business Review, July-August.

Kile, F. (2001). Insights into future international social stability. In P. Kopacek (ed.), Social Stability: The Challenge of Technology Development (SWIIS '01, IFAC), 45. Pergamon Press.

Laurillau, Y. and Nigay, L. (2002). Clover architecture for groupware. In $C S C W, 236-245$.

Reding, V. (2005). i2010: The European Commission's new programme to boost competitiveness in the ICT sector.

Thompson, K. (2008). The Networked Enterprise: Competing for the Future Through Virtual Enterprise Networks.

van Heck, E. and Vervest, P. (2007). Smart Business Networks: How the Network Wins. Commun. ACM, $50(6), 28-37$. 